\title{
SOLAR POWER PLANT AT PEAK LOAD BY SMART DISTRIBUTION
} SYSTEM

\author{
Babu Thomas ${ }^{1}$, Farzeen Faiz ${ }^{2}$, Abhilash Kumar M.D ${ }^{3}$, Athul Subran ${ }^{4}$, Anand $\mathbf{M}^{\mathbf{5}}$ \\ ${ }^{I}$ Assistant Professor [EEE], Dept. of Electrical and Electronics, Mar Athanasius College of Engineering, \\ Kothamangalam, India \\ ${ }^{2} U G$ Student [EEE], Dept. of Electrical and Electronics, Mar Athanasius College of Engineering, Kothamangalam, India \\ ${ }^{3} U G$ Student [EEE], Dept. of Electrical and Electronics, Mar Athanasius College of Engineering, Kothamangalam, India \\ ${ }^{4} U G$ Student [EEE], Dept. of Electrical and Electronics, Mar Athanasius College of Engineering, Kothamangalam, India \\ ${ }^{5} U G$ Student [EEE], Dept. of Electrical and Electronics, Mar Athanasius College of Engineering, Kothamangalam, India
}

\begin{abstract}
Solar power plants are designed according to the connected load. Generally, the peak load rarely reaches the connected load. Hence designing a system for connected load is uneconomical. System design can be based on the peak load by implementation of a Smart Distribution System (SDS) that saves cost of implementation of the PV system. SDS involves power allocation by automation. Here grid tied system is employed since solar power output is non-uniform. SDS employs a microcontroller that distributes the power based on a pre-defined time schedule, which also provides necessary safety features that protect system from failure and ensure proper distribution.
\end{abstract}

Keywords: Smart distribution system (SDS), Microcontroller, PV system $* * *$

\section{INTRODUCTION}

Over the last two decades, there have been two major events that led the world's industrialized nations to look at renewable energy sources as a supplement to providing the projected increase in energy demand. These include the world's energy crisis, effects of emissions from fossil fuelled power plants to both nature and mankind. Among a wide range of renewable energy projects in progress throughout the world, photovoltaic (PV) systems are the most promising as a future energy technology. Being cleaner, safer and abundant, solar PV generation provides a great scope for the future.

Solar power plants are generally designed based on the connected load. There are certain shortcomings in this type of design. Generally PV systems are designed at comparatively lower rating than the total load. Otherwise some means of storage is provided. Hence in design of system for connected load the chance of reverse flow of power from load to source is more. Also, this type of design makes the system installation expensive. As net metering facility is not available in all locations, the only solution to prevent reverse power flow is shutdown of the entire plant.

As concept of plant shutdown and thereby limiting the power generation is not an ideal one, proper utilization of available power is the feasible solution. By automating the power distribution it is possible to reduce the energy wastage. Hence peak load can be minimised. Installation of the PV system can be made cost-effective by designing it for the peak load rather than for connected load. Automation of power allocation is accomplished by a microcontroller based smart distribution system.

\section{DESIGNING A SMART DISTRIBUTION SYSTEM}

\subsection{Scope}

The designing of a solar power plant on the basis of peak load can be achieved in scenarios where the load requirement is cyclic, i.e. the pattern of load demand repeats its sequence on a daily basis so that power allocation can be automated.

\subsection{Load Calculation}

Like every power plant design determination of the net connected load is the first and foremost part of the project. Since peak load is regarded as the basis of the design, determination of peak load is to be done. Study on the load demands is conducted in half hourly basis to determine the pattern of load demand which is required for allocation and automation at later stages. The load pattern study is critical step as the allocation is done on the basis of time. 


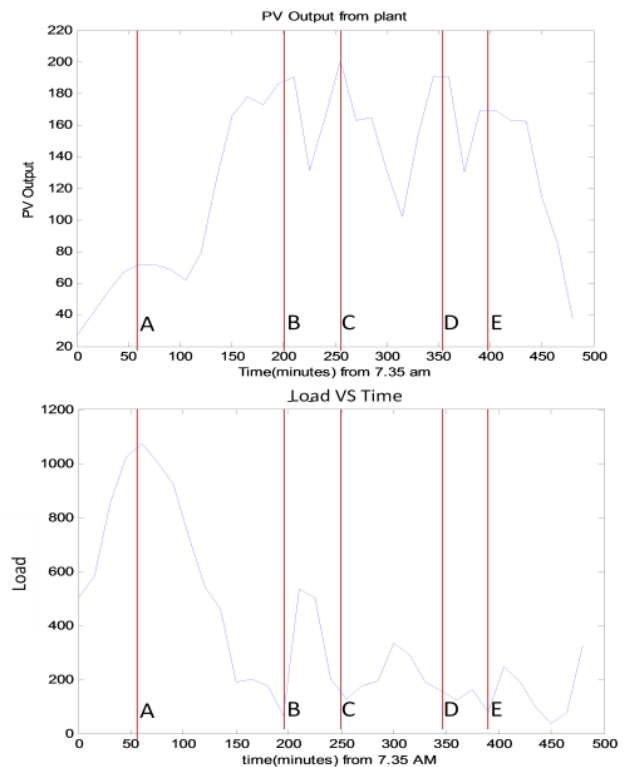

Fig 1 Generation and Load patters of an institution

Load and generation curves shown above explains the necessity of Smart distribution system. Load demand at the instant A is much greater than the generation. Hence suitable selection of some less important loads from there (load clipping) and distributing them at instants $\mathrm{B}$ and $\mathrm{C}$ where power generation is much higher than the load demand is possible. Also at instants D and E no extra load is available hence additional generated power need to be dissipated in some auxiliary loads generation. Thus proper sectoring and distribution of generated power is necessary that can be made with a smart distribution system which enables us to design a system at peak load.

\subsection{Power Allocation / Sectoring}

Allocation and Prioritising: After the load pattern is studied, the entire connected load is divided into sectors depending upon the time in which the load is connected. I.e. A industry where the cooling system is to be turned on at 12 noon, set of blowers and compressors has to be turned on at the same instant to accomplish this task so these machines are sectored in to one unit.

The same way as an entire industry is sectored based on time; machines that are turned on the same instance are brought under one sector. Further, the sectors were prioritised as per their role and function. As for any industry, the prime factor under consideration for setting priority is safety; the same is followed here also. This is critical in an instance the PV plant is running at full load and an emergency occurs the SDS will divert the power from other less prior sectors to the safety sector. Another important sector is the auxiliary sector in which loads that can be run at point of time are connected.

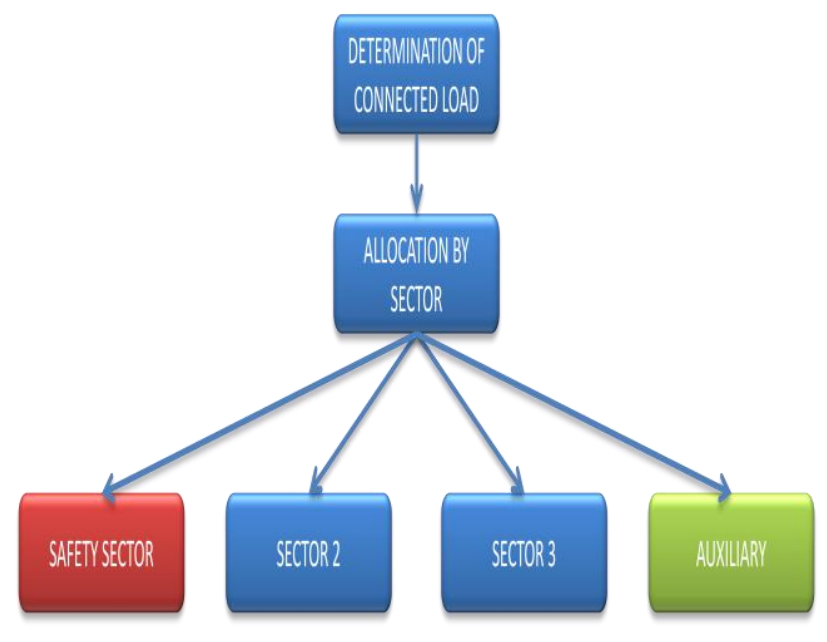

Fig 2 Process of power allocation

\subsection{Automation}

Automation of the system is done using a microcontroller. Power is allocated according to some predefined time slots to different sectors. In case, if power needs to be allotted to other sectors certain priorities are defined. If it is the case of safety sector, since this sector is of vital importance power to any trivial sector is temporarily cut off and safety sector is fed with the required power,. Power requirement in any other sector is considered by the controller only after checking the priority levels.

The problem of reverse power flow cannot be completely eliminated by designing at peak load. In some cases the generated power can still exceed the required load demand, thus causing reverse power flow. It is crucial in countries like India to prevent this as the net metering facility is absent here. Thus monitoring the power requirement of different sectors is essential.

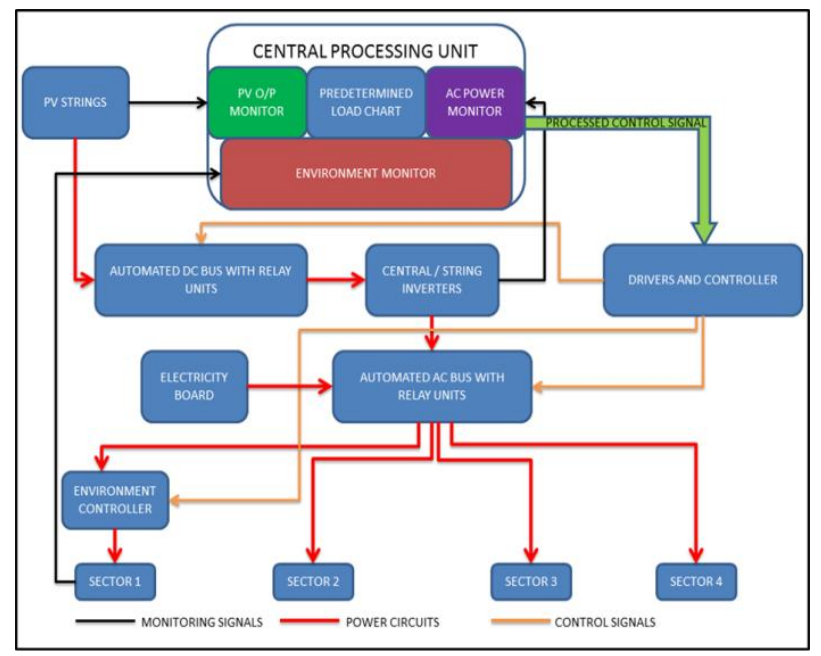

Fig 3 Schematic of smart distribution system 


\subsection{Environment Control}

A control unit is set for continuously monitoring and regulating all the lighting loads (lights, fans etc.) of the particular sector. Sensors are mounted to measure the light intensity and temperature of the sector which is then fed to the central processing unit that will generates the control signals to maintain a proper working environment.

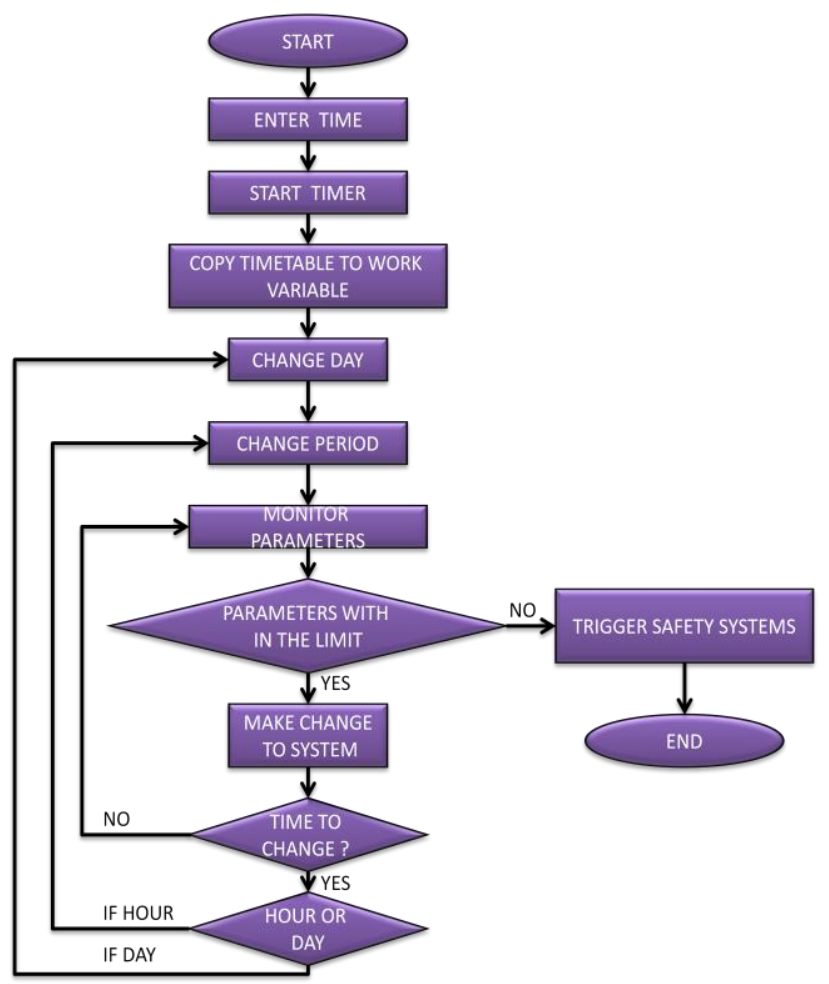

Fig 4 Flow chart of SDS Algorithm for an educational institution

\subsection{Complication in the System}

The issues that may arise in the design with peak load are,

i. Generated power can't meet the peak load demand.

ii. Generator power exceeding the peak load demand.

When the generated power can't meet the peak load, the remaining power can be met either from the EB or from a separate generator. For large industries a dedicated feeder to the site will be a better solution than that of a high capacity generator considering the initial and running costs of a generator. Since dedicated feeder only takes the initial capital investment, the extra power can be drawn from the EB in an economical way.

As already discussed, when the generated power exceeds the peak load reverse power flow will occur which is a matter of concern in areas where net metering doesn't exist. The burden of reverse power flow can be set aside by the efficient use of excess power generated. It is ensured by the auxiliary sector of the system. The auxiliary sector consists of different loads which need not to be allocated in a specified time slot. For example air conditioning, motor pumping etc. comes under such loads. The excess power is diverted into this auxiliary sector. That is, in case of reverse power flow, the excess power generated is utilised to meet various loads in the auxiliary sector.

Another alternative to drive out this issue is to control the output of the PV system. An automated SCB can be used for this function in place of an ordinary String Combiner Box (SCB) whose operation will be controlled by the microcontroller. The microcontroller continuously monitors the power output of the PV modules. When it exceeds the preset level, controller will disconnects an entire string from the SCB thereby reducing the PV output. An ordinary inverter used in the system is of the rating $450 \mathrm{~V}-600 \mathrm{~V}$ and $6 \mathrm{~A}$. So the minimum step of power is about $2.5 \mathrm{~kW}-3 \mathrm{~kW}$. In case if the extra load requirement falls between these steps, we should include the auxiliary sector in the system. Still, if this problem persists, then we go for a required power rated electric motor to simply dissipate this extra amount of power generated.

\section{CONCLUSIONS}

A power plant design based on peak load can be done along by the implementation of a smart distribution system to achieve significant savings in the area of energy consumption and usage. The difficulty of reverse flow of power can be completely uprooted by the introduction of a smart distribution system. Proper environment control will serve to monitor and reduce the usage of lighting loads of a sector, contributing to the concept of energy saving. Thus a solar PV plant with smart distribution technique can be used to obtain a reliable, cost effective, secure and an efficient system.

The load profile after implementing power allocation, showing that the peak demand has been considerably reduced

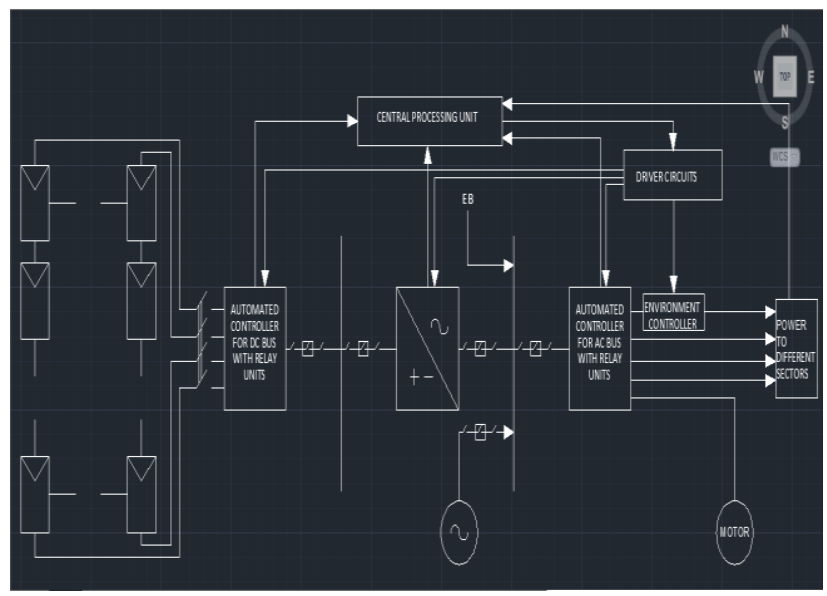

Fig 5 Layout of PV System with SDS 


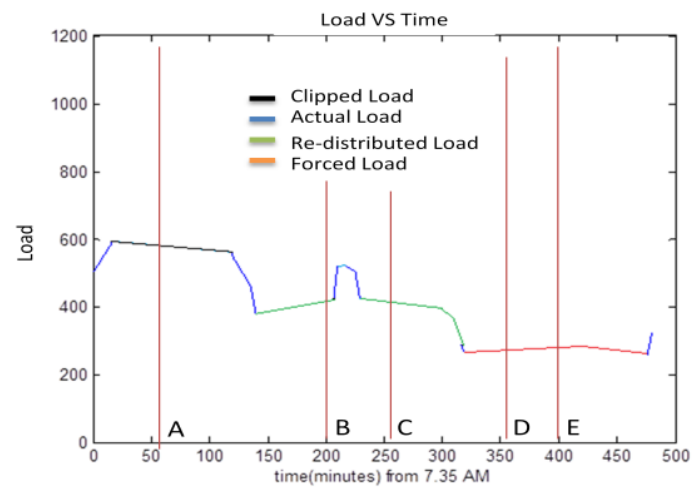

Fig 6 Load pattern after power allocation

\section{REFERENCES}

[1]. Noam Lior," Energy resources and use: The present situation and possible paths to the futures",2007 Elsevier Ltd, Department of Mechanical Engineering and Applied Mechanics, University of Pennsylvania.

[2]. Chetan Singh Solanki.," Solar Photovoltaic", Second Edition 2012

[3]. "NASA Radiation Data" https://eosweb.larc.nasa.gov.in .

[4]. "Solar Electric System Design", Operation and Installation $\odot 2009$ Washington State University Extension Energy Program.

[5]. "Grid-connected pv systems: system design guidelines", Sustainable Energy Industry Association of the Pacific Islands ,Issue 1 September 2012

[6]. ABM Musa, Md. Emran Chowdhury.," Smart Room: An Intelligent and Energy Conserving Living Environment.", Dept. of Computer Science \& Engineering, Bangladesh University of Engineering \& Technology, Dhaka 